\title{
Theory of Vibration in rubbing frictional materials
}

\author{
A. Bellutigi
}

Ricevuto il 18 Novembre 1964

Summary. -- The antisqueal problem of brake linings is a very complex one. As a result, the solutions which are obtained are always provisional or only approximate.

In fact, acoustic-vibrational problems involve "many degrees of freedom" which imply more "natural modes of motion", natural or resonant frequencies; it can be shown that an n-degree-of-freedom vibrational system has $n$ real natural frequencies.

However, in this Study, it is hoped that some new aspects of this problem have been sufficiently clarified.

It is evident that the "antinoise" problem, of which a general solution has been given, is not independent but closely related to other wear problems, mechanical strength and failures, which on closer examination require further developments.

Rinssunto. - Il problema di smorzare lo stridio di superficie interne frenanti, ̀̀ molto complesso. Le soluzioni che si sono ottennte sono state sempre provvisorie o approssimative.

In realtà, i problemi acustici-vibratorii implicano " molti gradi di libertà " i quali comprendono più " modi naturali di moto ", frequenze naturali o risonanti; si è potuto dimostrare che un sistema vibratorio ad $n$ gradi di libertà, ha $n$ frequenze naturali reali.

Tuttavia, in questa nota, si è sperato di avere sufficientemente chiarito alcuni nuovi aspetti di questo problema.

E evidente che il problema "dell'antirumore", del quale è stata data una soluzione generale, non è indipendente ma strettamente legato ad analoghi problemi, meccanici e non, i quali richiedono, per uno studio più approfondito, ulteriori sviluppi.

\section{TIIEORY of VIBRA'tion In Brakes}

1. - It is known that acoustic oscillations are manifest in the motion of "rubbing", subject to dry friction: aroustic energy of high intensity often accompanies vibration, "chattering slip", landom, periodic or" 
transient acoustic noises. These oscillations are generally, in a periodic condition, being therefore, only in the nodal points and are of variable width, usually detached from the harmonic form (superharmonic and subharmonic vibrations).

Many mechanical systems posses " non linear " characteristies, e.g.: simple pendulum, simple spring-mass system with snubber action, "stretched string with concentrated mass" or "belt friction system ". These systems involve a " non linear" charareteristic depending upon the dry friction between the mass and the moving belt, etc.

One distinguishing feature between linear and non-linear behaviour is the dependence of the period of the motion, in non-linear vibuation, on the amplitude (non-isochronous systems).

Sometimes the oseillations assume a "relaxation " or "discharging" form with a mpid decrease of elongation, by leaving the unstable equilibrium (static or dynamic), condition of finite length of time, allowing no relative speed, and therefore static friction roefficient $u$ during one part of the cycle.

A Coulomb-friction force generally results from the relative motion of two solid members held together under pressure: $F_{f}$ is directly proportional to the coefficient of friction $\mu$, the unit pressure $P_{n}$ between the surface and the area $S$ of contact is:

$$
F_{f}=\mu S P_{n} \text {. }
$$

The coefficient of friction $\mu$ is a function of the materials at the interface where relative motion occurs. In steady-state vibrations, the relative velocity becomes equal to zero, twice during each cycle.

Owing to a casual correlation between the vibratory motions in real dissipative cases and the recurrent friction, the roefficient $\mu$ must either be intrinsically dependent on time - with alternate law -, resulting in "forced" oscillations or otherwise the coefficient $\mu$ must provoke the vibratory stimulus depending on the relative motion, and therefore only indirectly dependent on time, with activation of self-excited vibrations.

The vibration of a mechanical system is self-induced if it results from conversion, within the system, of non-oseillatory excitation to oscillatory excitation. The oscillation of a system is forced (forced vibration), if the response is imposed by the excitation. If the excitation is periodic: and continuing, the oscillation is a steady-state. Considering the non linear equation of motion known as Van der Pol's equation, the principal feature of this self-excited system is in the damping term; for small 
displacements the damping is negative and for large displacement the damping is positive.

In a self-excited vibration the alternating force that sustains the motion is created by the motion itself; if the motions stops, the alternating force disappears. In a forced vibration the sustaining alternating force exists independent from the motion and persists when the vibratory motion is stopped.

Compressed air can be used to excite a structure in its fundamental mode of vibration (self-excited type).

In connection with such occurrences there are further theories, e.g.: " welded joints" of Bowden, the "electrostatic theory" of Schnurmann and Warlow-Davies, "friction self-excitation " of Rayleigh, the latter being the least contradicted.

The theory of "welded joints " or " stable bonds ", between solids in contact, as a consequence of high temperatures and high pressure, with the real surfaces in contact (limited in regard to those apparent), as a result of geometric disuniformity, gives an explanation of the disuniform character of the sliding friction.

These microweldings, formed by external forces in action, disappear with time. A friction contact alternatively static and kinetic (stick-slip) may be carried out in the relative discontinuous motion. A swing motion descends with physical characteristics inherent to "rubbing" solids, which reflect on the micromelting apart from the shear strength.

This theory, wherein one necessarily expects the possible discontinuity of the relative motion (which however, does not always appear) does not provide explanations as to the conditionally periodic character, always found in oscillations maintained by friction.

In effect, the hypothetical formation of "welded joints" does not necessurily afford periodical characters.

The electrostatic theory is inefficient if one considers electrical conductor solids in friction (e.g. drums).

As stated before, it seems evident that for the recurring of " selfexcitation" in "rubbing" borlies, the friction is required to vary non linearly with the speed, and that it admits a minimum, decreasing from the beginning of the motion. Conditions, which are expressed in analogical terms, are known for their strength associated with mechanical or electrical stable oscillator phenomena.

For this purpose, one must remember that in the motion of singledegree-of-freedom Vibrator (linear retourning), the dry friction strength expresses a characteristic function of the relative speed: $f\left(V_{0}-\dot{x}\right)$ 
(resultant from the uniform component $V_{o}$, and from the periodic $\dot{x}$ ), which can be represented with a complete differential equation in $\ddot{x}$, with the function $f$ as coefficient of the term in $x$.

If the upperterms of the series of expansion of Taylor of the same function $f\left(V_{0}-x\right)$ are neglected, (a proper choice of origin reference), the integral of this equation - with a real exponential factor, divergent or convergent according to the assumption $\approx 0-$, puts into evidence motions respectively unstable or "freestart". Then, if the assumption is null, the oscillator, formally conservative, does not go away from the quietness state, in every instant the kinetic energy integral being 0 . From that one excludes the possibility that in the oscillat or of elastic retourning (resistance 0 or linear resistance $\gtreqless 0$ ) the motion begins if there is a failure of periodic or steady activation energy. On the other hand, if one admits that for the divergency of the amplitude the retourning remaining linear, the higher terms of the series of expansion of $f$ obtaining importance - discarding the linearity premise of the strength - - it is possible to realize stable vibrations. If for "strength ", a binominal law decreasing with the increasing of $x$ is assumed (for small $\dot{x}$ ) and increasing with $\dot{x}$ (for great $x$ ) then Van der Pol's equation holds, which allows with approximation, a periodic integral, showing the stability of the oscillations whenever the law of dependence of strength — previously mentioned - occurs from $x$ (which is identified when the minimum condition is expressed).

One can attain from any other law of strength, analogical results, provided that it conforms with the conditions already stated. To integrate similar equations, one must proceed by means of graphs due to the lack of functional-integrals (Duffin, Kryloff, Ritz, etc.).

In such a way, one can present the attenuating influence of the damping strength of the self-exicted vibrations on the amplitude (to total damping), and also the infuence of pressure between "rubbing" bodies with even characteristies and relative speed (Dudley \& Swift). The abovementioned non linear and initially decreasing characteristic of the kinetic friction (coeff. $\mu$ ) has been examined under a physical aspect by Forrester. It is recognized that the contact area between solids in friction through which a material's continuity is realized, and therefore a strength motion, decreases with the increasing of speed, and - fundamentally - for the time factor influence on the stress/strain characteristics. In addition, the action of time will reflect the diffusion between materials in contact (forming alloys or sinters, materials harder than the components), and therefore the transitor bond strength made up of such sinters will generate friction. 
The increase of relative speed influences only initially the friction, whereas following the progressive adaptation of the surface contacts, attemuating the load concentration will remarkably reduce the influence of the arising thermic factors. If the "self-excitation " an sufficiently (but not necessarily) explain the vibratory friction, one must point out that the conditionally periodic vibrations exist, together with friction in dynamic conditions of the oscillator, not always suitable to insure self-excited motions.

Vibmations then originate when the dynamic specifications of the oscillator do not allow self-excited motions, which is a basic point to be kept in mind.

The causes for similar oscillations are revealed necessarily dependent on the intrinsic variability (of time) of the mutual action between solids in contact during " chemical treatment ". These intrinsic variabilities have structures due to thermical and chemical treatment, wear of the materials of discreet measure and not continuous, and consequent discontinuity in the motion, inhomogeneity effects, the whole being out of influence of the motion.

In conditions of relative motion, the process of "adiabatic distortion " takes place, which is limited around the actual contacts between the "rubbings", this process leading to cohesion and sliding.

Then the conditions of the motion controlled by other inherent factors of the oscillator and friction characteristics, are to allow that the process evolves towards mobility or solidity of the parts in contact.

Therefore, micromelting and microshearing through intermediary stages of fastness phase, particular cases of a general phenomenum of thermodynamic equilibrium, follow with individuality and consecutiveness. One finds therefore, distinct intrinsic physioal-chemical components of discontinuity (associated with coeff. $\mu$ ) of the friction-mechanical oscillations coherent with the kinetic friction between rubbing solids.

If in any frictiongrammes it appears that the vibrations maintained by friction have the natural frequency of the oseillator (which is favoured by this theory), then this frequency is excluded in general from the procedure of the characteristic of kinetic friction.

The "Friction Oscillator" in conclusion, is subjected to a complex exciting action, either by the components of limited energy or of great energy: Free Vibration, Forced Vibration, Resonauce, Self-excited Vibration, Stealystate, periodic - random - transient Vibration; linear, non linear, undamped, lamped, complex damping Vibrating Systems; Vibiat- 
ing Mass, spring Rate (suspension, tyre, roll); Vibration of vehicle suspension systems (bounce, pitch, roll), etc.

Some of these components, inherent to the many abovementioned frequency bands, are able to produce peak resonance, peak-to-peak amplitude, not on a simple frequency - as has been believed up to now - but on numerous natural frequencies on the vibuational system, which is primarily recognized.

2. - Starting point then is the motion of the vibrating lining in a form already elaborated upon in the first part of this study:

$$
x+(a L / m) \dot{x}+(b / m) x=\left(\mu_{0}+a V_{0}\right) L / m
$$

$m=$ mass per unit area,

$\mu_{0}=$ initial friction,

$E_{\mathrm{s}}-$ shear elastic constant,

$b=E_{s} / t$,

$a=$ change in $\mu$ with speed,

$L$ - load force per unit area,

$t=$ thickness of the lining.

The drum rubs against the lining with a speed $V_{o}$, lining moving at a speed $x$.

Assuming:

$$
\mu=\mu_{o}+a\left(V_{o}-\dot{x}\right)
$$

the integral of $[1]$ is placed in a convenient form (Basford-Twiss):

$$
x=A \exp .(-a L t / 2 m) \operatorname{sen}\left[\left(4 b m-a^{2} L^{2}\right)^{1 / 2} \cdot t / 2 m+B\right] .
$$

$A, B$, arbitrary constants, for the present purposes need not be evaluated.

From [2] one obtains:

$$
\dot{x}_{\max }=2 \pi \omega A \exp .(-a L / 8 m \omega)
$$

with

$$
\omega=\frac{\left(4 b m-a^{2} L^{2}\right)^{1 / 2}}{4 m} \text {, (natural frequency of lining) }
$$

and

$$
(\dot{E})_{t=0}=-2 E_{o} a L / 2 m
$$

where $E_{0}$ is local and transient excess of potential energy.

All things being equal, the "Probability of Noise" will be proportional to: $-a L / 2 \mathrm{~m}$. 
From [2] one deduces that an initial displacement $x$ in the lining can grow into an acoustical vibration (starting squeal) only if the following condition occurs: $E_{s}>t L^{2}\left(a^{2} / 4 m\right)$.

If $a<0$, one has self-reinforeing: if $a>0$, one has self-damping vibrations.

Self-reinforcing always becomes more accentuated according to $E_{s} / t$, having low medium and high values, with predominant "stick vibrations" with the increasing of $E_{s} / t$.

The joint-effects of $d \mu / d v$ and $E_{s} / t$, in determining vibration, reflect back differently on the form of vibration - according to the region of low - medium or high shear elastic mod./thickness of lining.

Then, the fundamental action of bond-shearing friction mod. $E_{s}$ on which the noise depends, may be exceeded at a given threshold level. The noise may be eliminated by conferring to the brake lining:

$$
E_{s}<t a^{2} L^{2} / 4 m \text {. }
$$

The natural frequency of the drum and that of the vehicle support co-vibratings have been left out in this theory (suspension springs, coupling springs, anchorages, dampers, inertial excitations, etc. able to rebound on the friction vibrations). The repercussions of the co-vibratings are frequently predominant, and this may be shown by the following considerations:

continuing from the simplest position of induced forced vibrations and having the forcing frequency $\Omega$ :

$$
\dot{x}+(a L / m) \dot{x}+(b / m) x=L\left(\mu_{o}+a V_{o}\right) / m+A \operatorname{sen} \Omega t
$$
(equation of self-exicted and forced vibrations).

With an inertial excitation (frequency and impedance remaining) the coefficient of sen $\Omega t$ will be $A \Omega^{2}$ instead of $A$, that is to say a sonic intensity or a sound wave $\Gamma_{\Omega}<\Gamma$, ratio $\frac{\Gamma \Omega}{T}=\Omega^{4}$, because:

$$
\Gamma=2 c \varrho_{\circ} \pi^{2} f^{2} A^{2}, \quad \Gamma_{\Omega}=2 c \varrho_{\circ} \pi^{2} f^{2} A^{2} \Omega^{4} \text { watts } / m^{2} \quad \text { where }
$$

$f=$ frequency,

$c \varrho_{0}=$ characteristic impedance of the acoustical medium,

$Q=$ densities,

$c=$ wave's velocities.

The remarkable ratio $\frac{T_{O}}{\Gamma}$ induces deleterious stress-amplifications and failures and acoustical fatigue, owing to the noisier energy which increases wear. 
The solution to $\left[1^{\prime}\right]$ consists, it is noted, of a "complementary function " plus a "particular integral».

The complementary function has the following ehancteristies:

$$
a=-a L / 2 m \pm\left(a^{2} L^{2}-4 E_{s} / t\right)^{1 / 2} / 2 m
$$

which according to $a^{2} L^{2} \geq 4 E_{s} / t$ gives standard results, as in the case of " (lampers" (over, critical, underdamped).

In the first case of the abovementioned disequality, since

$$
\left(a^{2} / L^{2}-1 E_{s} / t\right)^{1 / 2} / 2 m<a L / m,
$$

the roots of $\alpha$ are negative $(-\gamma,-\delta)$, and since $\alpha=X, x=0$ for $t-0$, one has:

$$
x=X e^{-\gamma^{\prime} t} /(\delta-\gamma)+X e^{-\delta t} /(\gamma-\delta)
$$

In the second case (two roots of complementary function are equal):

$$
E_{s}=1 / 4 a^{2} L^{2} t
$$

In the third case (conditions of vibrational insurgency):

$$
x=A a^{-n t} P \operatorname{sen}(m t+\psi)
$$

where $n, m, P, \psi$ are constants deducible from the initial conditions.

The periodic time of the motion becomes:

$$
T=\frac{4 \pi}{\left(4 E_{\mathrm{s}} / t-a^{2} L^{2}\right)^{1 / 2}} .
$$

The particular integral is obtained if

$$
x=B \operatorname{sen} \Omega t \text { (disturbing force) }+L / b\left(\mu_{0}+a V_{o}\right) \text { is considered. }
$$

Putting these values into equation $\left[1^{\prime}\right]$, one obtains:

$$
\text { sen } \Omega t\left(-\Omega^{2} B+b B / m-A\right)+k \Omega B \cos \Omega t=0 \text {. }
$$

For

$$
\Omega=\pi / 2, \quad B=\frac{A}{b / m-\Omega^{2}}
$$

Therefore, the displacement $x$ becomes:

$x=e^{-n t} \varrho \operatorname{sen}(m t+\psi)+A \operatorname{sen} \Omega t \cdot\left(b / m-\Omega^{2}\right)^{-1}+L t\left(\mu_{o}+a V_{o}\right) / E_{s}$ with evident resonance for $\Omega=\sqrt{E_{o} / m t}$.

The condition for the elimination of noise:

$$
E_{s}<a^{2} L^{2} t / 4 m,
$$


is no longer sufficient; it is necessary to add to it the condition:

$$
E_{s} / m t \gtrless \Omega_{i}^{2} \text {. }
$$

Therefore, it is necessary to recognize the amplitude and frequencies of the foreed vibrations $\Omega_{i}$ firstly, comparable with the Fourier components, which are multiplied by the "amplificator factor ":

$$
\left(b / m-\Omega_{i}^{2}\right)^{-1}
$$

and to avoid for that reason that the values $E_{s} / m t$ coincide with those relative to $\Omega_{i}{ }^{2}$.

In other words, one adds the particular outside frequency acting on the brake lining with individual vibratory, which induces resonant-peaks, with intensity up to $\infty$ (according to the progressively decreasing values of $a L / m)$. Or corresponding with the frequency ratio $1=r=\Omega / \omega$, variable parameter a $L / m$, decreasing till 0 , the dynamic Magnifier $M=\infty$, after $M$ decreases again from $\infty$ or from the relative max. $r=\Omega / \omega=1$, up to vilues $<1$.

This result, analogous to that with the motion of a single vehicle on an undulating road, persists if the periodic sinusoidal function, relative to the forced vibration has a phase $\varphi$, and the quantity $B$ becomes:

$$
B=A\left[\left(\omega^{2}-\Omega^{2}\right)+a^{2} L^{2} / m^{2}\right]^{-1 / 2} .
$$

For $\Omega \rightarrow 0, B=A \mid \overline{m t / E_{s}}$, and for $\Omega<E_{\delta} / t, B \rightarrow 0$.

The examination of function $B$ is facilitated when one observes that the maximum value of the amplitude $B_{\max }$ of the forced vibration (which is obtained with $d / d \Omega-0$ ), is:

$$
B_{\max }=2 m A / a L\left(4 b m-a^{2} L^{2} / m^{2}\right)^{1 / 2} .
$$

The system is said to resonate with the corresponding frequency $f_{r}=\Omega_{m} / 2 \pi$. The dynamie Magnifier $N I$ is in this case:

$$
M=\left[\left(1-r^{2}\right)^{2}+r^{2} a^{2} L^{2} t / m E_{s}\right]^{-1 / 2}
$$

with $r$ frequency ratio.

The vibrational displacement for the forced motion is expressed in the following closed forms:

$x=-\operatorname{sen} \varphi \operatorname{sen}(\Omega t+\varphi) m A / a L \Omega, \quad \operatorname{tg} \varphi=-a L \Omega_{n}\left(E_{\delta} / t-m \Omega^{2}\right)$,

particularly when the velocity of the motion is of more interest or significance than the displacement:

$$
x=-\operatorname{sen} \varphi \cos (\Omega+\varphi) m A / a L \Omega .
$$


Usually, the coeflicient of the sinusoidal term $A$ in the forced vibration, which represents the max. of the inciter force, is considered as a constant amplitude, inclependent from the frequency. Since this is not always true, especially if the inciter or exciting foree is unbalanced, then the maximum of $A$ varies with the square of the frequency, and instead of $A$, one has $A \Omega^{2}$ and $B_{i}=-m A / a L \Omega=r^{2} B$.

In conclusion, having demonstrated that for $\Omega^{2}=b / m$ the forced vibrations fall into resonance, one las to modify the "limitations" $E_{s} \gtreqless a L t / 2 m$, of incipient vibratory, obtained from the specific lining contribution.

It is indicated too that, when developing an "antinoises lining", it is necessary to recognize above all, according to the types of vehicles on which they will be applied, the frequency bands of the assembly, of the single parts and of those in a position to vibrate much more.

The shear elastic moduli $E_{s}$ of these lining-systems (having distributed mass and elasticity) have values outside those of the spectrum-limits.

In the following formula the antinoise conditions are summed up, (with an exception made for improbable " white noises "):

$$
E_{s} / t \neq \Omega_{i}^{2}<a^{2} L-/ 4 m \text {. }
$$

Finally, no "free vibrational zones " exist, which are distinctly separate from the "unfree zones" or "pseudofree zones". However, they could exist if $E_{s} / t \neq \Omega_{i}{ }^{2}$ (frequencies of the vehicle identified in advance). Otherwise the limitation < would not be of value, free and forced vibrations, resonant or not, could arise on all sides; same lining can be acoustic in one vehicle and not in another.

Moreover, the probability of noise in the " unfree zone " can be reinforced, etc., being daily experienced.

A single lining may be quiet on one brake and noisy on a brake of different design. Linings with substantially equal may be very different in their tendency to noise. The types of vibration and its occurrence are greatly influeneed by the dynamical properties of the mechanical system involved.

The really vibrational phenomenon of "rubbing-bodies" in relative motion, of interfacial contact, gives numerous casues of squeal, and camnot be left out of consideration from the repercussions of the "brake-systems" and relative " mountings".

Any antisqueal lining (or adsorber) must always be considered relative to its brake assembly, and to its vibrational couplings. Among 
these, the lining-drum resonance (ovaling drum vibrations), the shoe vibrations, and other inadequate fittings, lining-shoe, defective brake shoes, pivot distortions, etc. are to be remembered.

It has been observed that the brake squeal sometimes occurs when the natural frequency of a brake shoe is near one of the natural frequency of the brake drum.

In the first part of this study it has been hoped to have shown a "rating " of "deadening selective linings "; here it has been demonstrated that it is possible to manufacture a wholly antisqueal material.

The conclusions that have been drawn seem relative to excessively high mathematical schemes; however, they are still less than those of preceding authors.

The more concrete vibrating systems, with more degrees of liberty, have been the main concern of this study; e.g.: mass moving up and down together with different amplitudes (one mass moving up when the other moves down), and the results do not change.

The equations of motion of mass $w_{1}$ and $w_{2}$ (elastic vertical coupling brake linings-bearings) are:

$$
\begin{aligned}
& \ddot{x}_{1}+k_{1} \dot{x}+\omega_{1}^{2} x+\omega_{12}^{2}\left(x_{1}-x_{2}\right)=0, \quad \ddot{x}_{2}+\omega_{2}^{2}\left(x_{2}-x_{1}\right)=0 \\
& k=c g / \omega_{1}, \quad \omega_{1}^{2}=k_{1} g / w_{1}, \quad \omega_{2}^{2}=k_{2} g / w_{2}, \quad \omega_{12}^{2}=k_{2} g / w_{1} .
\end{aligned}
$$

Placed as usual, so that the displacements $x_{1}$ and $x_{2}$ will vary sinusoidally with time:

$$
x_{2}=a_{2} \operatorname{sen}\left(\Omega_{2} t+\varphi\right), \quad x_{1}=a_{1} \operatorname{sen} \Omega_{1} t,
$$

$\varphi$ (phase angle for the motion $\alpha_{2}$ ); $\phi \neq 0$ is an essential condition since it must not be assumed that this motion is in phase with $x_{1}$.

Substituting the values $x_{1}$ and $x_{2}$ in equation-system, two other equations are derived, and since these equations must hold for all values of $t, t$ may be put equal to 0 .

The first equation becomes:

Becanse:

$$
-\omega_{12}^{2} a_{2} \operatorname{sen} \varphi=0 .
$$

$$
\omega_{12} \neq 0, \quad a_{2} \neq 0, \quad \operatorname{sen} \varphi=0, \pi, 2 \pi, \ldots
$$

and the preceding equation may be written in general as: $\operatorname{sen} \Omega_{1} t\left[-a_{1} \Omega^{2}+k \Omega_{1} a_{1}+a_{1}\left(\omega_{1}^{2}+\omega_{2}^{2}\right)\right]-\omega_{12}^{2} a_{2} \operatorname{sen}\left(\Omega_{2} t+\varphi\right)=0$.

Since the coefficients of the sen terms, in this equation, are independent of time, it follows that the equation can be satisfied for all values of $t$, only if $\Omega_{1}=\Omega_{2}=: \Omega$. 
With simple transformations, an equation can be obtained so that the ratio $a_{1} / a_{2}$ from the two equations separately, and equaling the two parts gives the values of $\Omega$ :

$$
\Omega^{4}+k \Omega^{3}+\omega_{1}^{2} \Omega^{2}+k \omega_{2}^{2} \Omega+\omega_{2}^{2}\left(\omega_{1}^{2}+\omega_{2}^{2}-\omega_{12}^{2}\right)=0 .
$$

There are four independent modes of vibration: the lower frequency can, approximately, approach the frequency of the mass $w_{2}\left(w_{2} \gg w_{1}\right)$ if $w_{1} \rightarrow 0$, and the higher frequency is approximately equal to that of the mass $w_{1}$, if the mass $w_{2}$ were held fixed.

If $\omega_{2}^{2} / \omega_{1}^{2}=k_{2} w_{1} / k$ and $w_{2} \rightarrow 0\left(w_{2} \gg w_{1}\right.$ real situation $)$, from the first equation the following occurs: $\omega_{12}^{2}, \omega_{2}{ }^{2} / \omega_{1}{ }^{2} \rightarrow 0$, and one can draw:

$$
\left(\omega_{2}^{2}-\Omega^{2}\right)\left[\Omega^{2} / \omega_{1}^{2}+k \Omega / \omega_{12}+\left(\omega_{1}^{2}+\omega_{2}^{2}\right) / \omega_{1}^{2}\right]=0
$$

at the limit one has:

$$
O=\omega_{2}=\mid \overline{E_{s} / t}
$$

or: $\frac{\Omega^{2}}{\omega_{1}}+k \frac{\Omega}{\omega_{1}^{2}}+1=0$, which is the usual condition $\frac{E_{s}}{t}<\frac{a^{2} L^{2}}{m}$.

Therefore, in the ranges $E_{s} / t<a^{-2} L^{2} / m$, it is necessary to recognize, given a certain thickness $t$ of the lining, which frequency coincided with $E_{s} / t$, to operate above all: $E_{s} \neq \Omega^{2} t$.

With these conclusions one must reflect on the "industrial deafness ", "safety engineers", "industrial and insurance medicine" (otology). Is besides the elasticity $E_{s}$ one has to keep into account in the structure of the lining, the visco-elastic quality (as in reality), dissipative forces are then assumed to be proportional to the particle velocity.

In such a case, another term $\partial^{3} y / \partial x^{2} \partial t$ has to be added for the equation for a wave in elastic medium:

$$
\frac{\partial_{2} y}{\partial t^{2}}=c^{2} \frac{\partial^{2} y}{\partial x^{2}}, \quad \varrho \frac{\partial^{2} y}{\partial t^{2}}=M \frac{\partial^{2} y}{\partial x^{2}}, \quad c=1 M / 0 \quad \text { where }
$$

$y=$ particle displicement,

$x=$ distance in direction of propadation,

$c=$ wave velocity.

Assuming then a solution, such as $y=A \exp .[-i(\omega t+k x)]$ this is substituted by the preceding equation.

However, the modulus and " dissipation coefficient " camnot ve taken as eonstants, since both the elastic and loss properties of the material vary widely with frequency. The complex longitudinal bulk wave mo- 
dulus $M^{\circ}$ is related to the complex bulk compression modulus $K^{\circ}$, and the complex shear modulus $G^{\circ}, M^{\circ}=K^{\circ}+4 / 3 G^{\circ}$.

These complex moduli are related to the elastic and loss moduli:

$$
K^{\circ}=K^{\prime}+i K^{\prime \prime}, \quad G^{0}=G^{\prime}+i G^{\prime \prime} .
$$

The displacement is:

$$
y=y_{\circ} \exp .[i \omega t-(\alpha+i \omega / c) x], \quad-\omega^{2} \varrho=M^{\circ}(\alpha+i \omega / c)^{2},
$$

from which real and imaginary parts $M^{\prime}$ and $M^{\prime \prime}$ are:

$$
M^{\prime}=\varrho c^{2}\left(1-r^{2}\right) /\left(1+r^{2}\right)^{2}, \quad M I^{\prime \prime}=2 \varrho c^{2} r\left(1+r^{2}\right)^{2},
$$

$\alpha=$ attenuation in nepers/cm.

$r=\alpha c / \omega$,

$c=$ phase velocity.

In the same way it may be derived for $G^{\prime}, G^{\prime \prime}, E^{\prime}, E^{\prime \prime}$, which are the relationships for obtaining the real and imaginary distortional wave constants.

When the attenuation for wavelength is small $r^{2}$ can (to a first approximation) be neglected in the above equations, the elastic modulus taken simply as $\varrho c^{2}$ and the loss modulus as $2 \varrho c^{3} \alpha / \omega$, referring back to the cases already studied.

It must be remembered that brake squeal frequencies cover a range from 2000 to $50000 \mathrm{cps}$. (range of annoyance $=10000$ to $15000 \mathrm{cps}$.). 